\title{
The Virtual Prototype Design of the Robot for Coal Mine Automatic Installation Rack Bolt
}

\author{
Fengju $\mathrm{Hu}^{1, \mathrm{a}}$, Jixin $\mathrm{Liu}^{2, \mathrm{~b}}$, Chengguo Zong ${ }^{3, \mathrm{c}}$ and Yungui $\mathrm{Li}^{4, \mathrm{~d}}$ \\ 1,2,3,4 Qingdao Huanghai University, P.R.China \\ asdhfju@sina.com, bsdljxin@sina.com, c $719362477 @ q q . c o m,{ }^{d} 1873157179 @ q q . c o m$
}

\begin{abstract}
The robot for coal mine automatic installation rock bolt can realize the automatic installation of anchor cable equipment in the mine. According to characteristics of robot operation in down hole, especially on its mobile function, anchor device and rack bolt operation device function was analyzed, and the crawler walking mechanism was studied. Based on the study, active arm, horizontal moving guide rail, automatic installation of anchor cable, anchor drilling institutions and other major institutions were designed and installed on the robot. Using 3D virtual prototype technology, modeling of key parts, the 3D virtual prototype is design.
\end{abstract}

Keywords: Robot, Rack bolt, Crawler, Virtual prototype

\section{Introduction}

With the continuous development of coal technology, the rapid development of fully mechanized coal mining has also revealed a serious shortage of fully mechanized mining, which brings great pressure to the production of the working face. There are two main factors that affect the rapid excavation: first, the speed of cutting coal, and the two is the installation speed of bolt and cable support. At present, the bolt and anchor bolt support technology in fully mechanized tunneling construction mainly adopts the single bolt drilling machine, the mechanization degree is very low, which seriously restricts the driving speed. The mechanization of anchor cable support is one of the key technologies to solve the problem of the anchor cable supporting efficiency and reduce the labor intensity. Therefore, it is very important to further improve the efficiency of anchor bolt and anchor cable support in coal industry.

At present, the anchor cable support machinery mainly uses the anchor drill, the hydraulic drill car, the dig and anchor integration machine and so on. The development of these devices, to a certain extent, to ease the contradiction between the speed and the speed of the excavation, improve efficiency, but not fundamentally solve the problem of low efficiency of anchor cable support, labor intensity. Therefore, it is an effective way to improve the efficiency and reduce the labor intensity of the bolt and anchor cable support equipment.

\section{The Design of the Robot}

The Design of the Mobile Robot. Walking device of construction machinery includes wheeled walking device and crawler type walking device.

Wheeled walking device consists of frame, axles, wheels and suspension components, main function and its characteristics are as follows.

(1) Supporting the total gravity of the whole machine.

(2) To accept the torque transmitted by the engine through the transmission system, and through the driving wheel and the adhesion between the ground, the driving force, to ensure the normal operation of the machine, the operation.

(3) The various kinds of reaction force and the moment formed by the transmission and supporting of the road surface

(4) As much as possible to ease the impact and vibration caused by the body, to ensure the smooth running of machinery. 
The crawler type walking device has the following characteristics compared with the wheeled walking device:

(1) Caterpillar driving wheel winding device and the rolling track only not on the ground, the weight of the whole machine by roller pressure in the multi track plate, all the weight was attached weight, adhesion track grousers and much better than the tire tread, so crawler traction machine the strength should be much larger than the wheel.

(2) Compared with the same power wheeled machinery, the track bearing surface, the ground pressure is small, so the depth of subsidence on soft soil is small, so the rolling resistance, is conducive to higher traction.

(3) The track is not afraid of mechanical damage, such as cut, cut, etc..

(4)Track pin, pin sets pair in use to wear, to have tension adjusting track tightness, it also plays a buffer role.

According to the characteristics of the crawler type walking device and the wheel walking device, the working requirement of the automatic installation of the anchor rod robot is compared, and the crawler type walking device is selected.

The Design of the Belt Working Device. The working mechanism of the rock bolt drilling machine is composed of a number of functional modules, and complete the assigned task under the coordination of each function module. The general working mechanism function module comprises a power module, a drive module, an executive module, a control module, a connecting module and a supporting module. The power module is the power source of working mechanism; the transmission module is made module and the motion transmission to the intermediate device to execute module; execution module is directly completed part of the task system; the control module is a control module and a control module, in order to make the power module, drive module, execution module and coordinated operation, accurate completion device of the operating mechanism; the connecting module each module (or modules components) are connected, forming a complete mechanical system; the supporting module is to link the working mechanism and the bearing is fixed, positioning or guiding role of working mechanism. In order to meet the functional requirements and constraints of the target mechanism, and bring convenience to the analysis and design, it is necessary to adjust the division of the function modules. According to the actual situation, through the function module supplement, the analysis or the merger and so on method, determines the suitable target organization's function module division plan.

The Design of the Anchor Cable Working Device. The anchor cable installation mechanism comprises two sliding mechanisms and a fixing mechanism, and the sliding mechanism is mainly composed of a rubber wheel, a lead screw, a shaft, a bearing seat and a supporting frame. The fixing mechanism is composed of a rubber wheel, a shaft, a shaft coupling, a motor and a supporting frame. Its working principle is to turn the handle to screw the rotary motion into linear motion, driven sliding mechanism move toward the center, when reaching a certain position using the function of self-locking screw fixation, and joint action and fixing mechanism, three rubber wheel clamping cable in motor drive under the rubber wheel fixing mechanism to rotate in addition to the two wheel drive cable attached to the upward movement, reached the purpose of cable installation.

\section{The Composition of the Robot}

Automatic installation of anchor robot is mainly composed of crawler type walking mechanism, a chassis, a hydraulic cylinder, active arm, horizontal moving guide rail, a driven arm, hydraulic station, chassis steering, automatic installation mechanism, cable anchor drilling mechanism etc.. Power supply and hydraulic system of the system, the use of drilling and anchor cable automatic installation of the use of coal mine underground tunnel gas source.

The Chassis Structure of the Robot. The chassis structure is composed of a steering mechanism and a supporting platform, which is used for installing and executing the structure, the hydraulic station, the control box and the power box. A parallel four bar mechanism is composed of an active big arm and a driven arm, which is used for supporting the steering mechanism of the sliding guide rail and the drilling and installation mechanism of the anchor cable. 
The Installation Mechanism of Anchor Cable. The anchor cable drilling mechanism has the advantages of simple structure, high efficiency and high efficiency. The anchor cable installation mechanism adopts the bionic design, which is composed of an upper and a lower clamping arms and a telescopic arm. Upper and lower clamping hand can imitate human into the cable action to achieve the installation of anchor cable. The upper and lower motion of the clamping hand is realized by the telescopic arm. In order to realize the drilling and the anchor cable installation of the side slope and the inclined plane of the coal mine tunnel, the horizontal guide rail structure is designed, and the angle of the horizontal guide rail is adjusted by the rotating mechanism to adapt to the different position of the drilling hole. In the cable installation is completed, the cable installation mechanism installed on the cable stretching equipment, then cable tensioning operation.

The Composition and Structure of the Walking Device. The crawler type walking device is mainly composed of a driving wheel, a guide wheel, a supporting wheel, a supporting wheel, a track, a tensioning device and a frame. The walking device is the role of supporting the whole weight, and through the rotation of the drive wheel tracked into the machine on the ground running, its main role is to realize the automatic installation of anchor robot walking underground, produced by hydraulic motor power walking. Crawler type walking device has a support area, the ground pressure is small, not easy to slip, traction and other features, the crawler has certain climbing and obstacle clearing ability, suitable for the operation of underground environment requirements. As shown in fig. 1'.

(1) To the ground, the gravity of the crawler is used to machine and ensure the machine produce enough driving force to device.

(2) The driving wheel is the power of will drive system to track, to produce the driving force of the vehicle movement.

(3) Gravity roller used for supporting machine. Rolling on crawler caterpillar track section, it is used for clamping the treads, prevent track horizontal slip derailment, and to drive the track in the ground to the upper sliding.

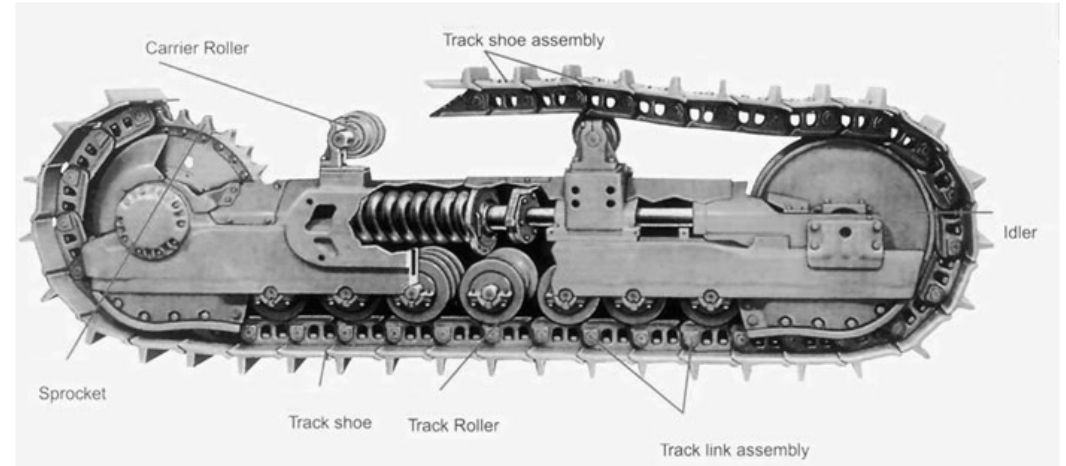

Fig. 1 Automatic installation bolt three-dimensional virtual prototype of the robot

(4) The roller is used to hold the upper guide between the wheel and the drive wheels crawler, prevent crawler prolapse is too large, in order to reduce the vibration when the crawler movement jumping phenomenon.

(5) The function of guide wheel is a winding correctly guide track, at the same time using the tensioning device making guide wheel to adjust the crawler tension.

Anchor device, the structure and composition of homework. The crawler type walking Jumbolter design should follow the following principles: monomer hydraulic jumbolter for key components, the adaptability of hydraulic jumbolter stronger; Machine with hydraulic jumbolter should have good compatibility, mutual influence their respective functions, do not interfere; Design of anchor drilling machine should be able to meet the anchor bolt hole, machine shift a request; Hydraulic jumbolter hydraulic circuit connection is simple; Unit, simple in structure, convenient in deep drilling a fuselage short.

Anchor drilling machine is mainly composed of main frame skin protection device, a propulsion device double lift propulsion mechanism of the hydraulic motor and hydraulic motor assist device. 


\section{UG modeling of key parts.}

The main components of the structure. Walking device of construction machinery includes wheeled walking device and crawler type walking device.

(1) Rotating arm by plates welded together, through the range of hydraulic cylinder, spin arm driving arm connected with the machine. When hydraulic cylinder luffing forward rotating arm rotating around a fixed on the driving arm protection makes the whole device rotate 90 degrees out forward position. Along with the adjustment of the hydraulic cylinder, telescopic arm bracket is in a state of perpendicular to the ground, and through the forward rotation, to ensure the out a certain distance, so that the rig can work within the operational areas required. When the rotating arm backward rotation, make the whole device backward, so that they don't hinder the driving arm work, and reduced the height of a complete set of equipment, reduce the space of the. As shown in fig. 2.

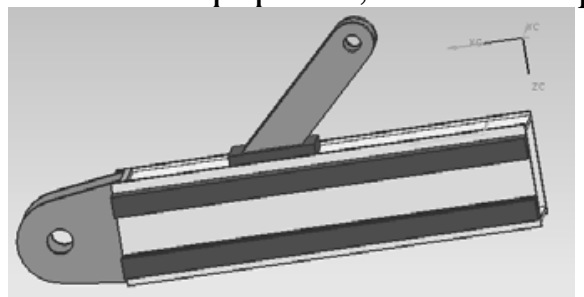

Fig. 2 Rotary arm

(2) Telescopic hydraulic cylinder 1. The DG model for vehicle hydraulic cylinder. Earrings are connected to the rotating arm, by the edge of the other earrings are connected to the telescopic boom, when the hydraulic cylinder and stretched forth, push the telescopic arm forward out of a certain distance, to keep a certain distance, before allowing jumbolter to work within the area of required.

(3) Telescopic crane. By the steel plate welding and become, when the need to arm elongation, by content with rotating arm of the telescopic crane scale of telescopic hydraulic cylinder to drive, and stretched forth on the telescopic arm has a few support the slider below, when the telescopic crane telescopic hydraulic cylinder pushing forward movement can slide down the slide forward. Make the complete scale effect. After its role mainly in the rotating arm rotation, on the basis of the forward slip distance, in order to realize long-distance operation. As shown in fig. 3.

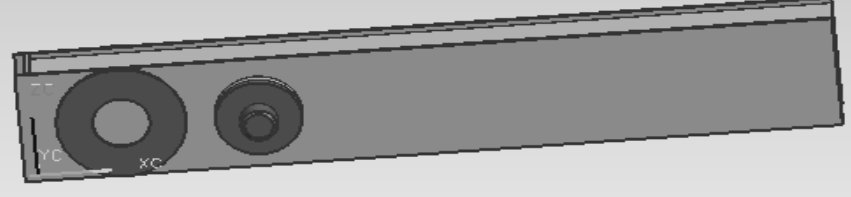

Fig.3 Telescopic arm

(4) Adjust the hydraulic cylinder: the DG model for vehicle hydraulic cylinder. When installation to make the adjustment of the hydraulic cylinder of the original state in the piston out, keeping support arm and rotating arm 90 degrees of the original state. When need to adjust the bracket and the relative position of telescopic boom, would be controlled by telescopic hydraulic cylinder, in order to obtain a suitable location. Adjust the hydraulic cylinder on your right earrings on the slider mounted on the rotating arm, left earrings are connected to the bracket, keep the two components are 90 degrees of the original vertical state. When the telescopic arm extended forward, also drive the adjustment of the hydraulic cylinder slides along the rotating arm moving forward, so as to keep the support arm, telescopic boom, adjust the hydraulic cylinder position remains the same in the original state.

(5) The bracket: by the steel plate welded together. Bracket are connected to the telescopic arm, and by adjusting the hydraulic cylinder, bracket and the telescopic boom in perpendicular to the original state, when the telescopic arm parallel to the ground has a tiny error, also is the support arm is vertical to the ground, can through the adjustment of the hydraulic cylinder, the support arm in vertical with the ground. Bracket support is the main purpose of drilling operation platform, and to ensure that the whole platform horizontal position in space, in order to make sure the rig drilling of vertical. As shown in fig. 4. 


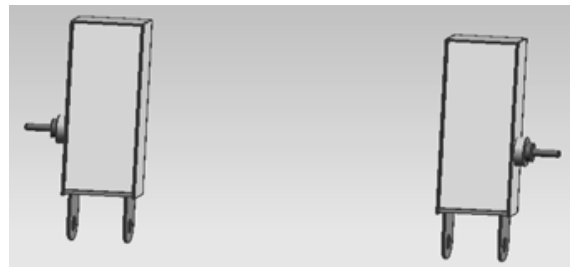

Fig.4 Support arm

(6) Drill supporting platform. Welding on the top of the bracket. On the platform of the end face, set up the slide guide rail, and platform together. In the interior of the platform, set up the connection of the hydraulic cylinder bearing, and place the telescopic hydraulic cylinder, its and even on the slide rail connected to the support of the slider, move the slider to the left and right sides of the control. As shown in fig. 5.

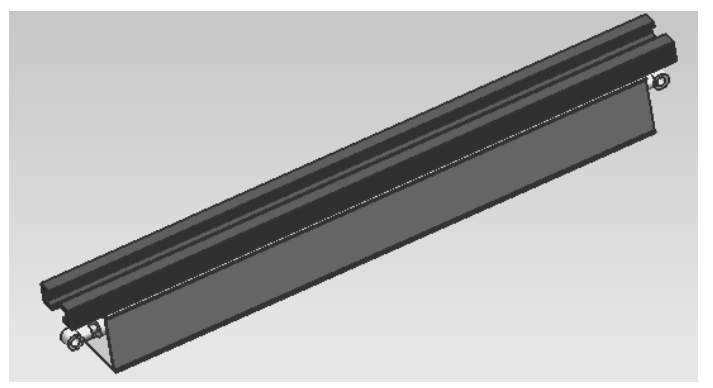

Fig.5 Drilling rig supporting platform

(7) Telescopic hydraulic cylinder 2. The DG model for vehicle hydraulic cylinder, a total of two, placed within the rig support platform and the lateral earrings and support the slider are linked together, when the telescopic hydraulic cylinder telescopic support on both sides of the slider to move.

(8) To support the slider. Support the slider in the rig support platform on the slippery course of moving. When in the original state, two supporting the slider is located in the center of the slide rail, on both sides of the slider to move the largest distance. When two slider in the biggest stretch out position, drill horizontal distance to provisions of the dimension of drilling hole, satisfy the requirement of the bolt hole arrangement. In supporting the front end of the slider profile, set up the slippery course, jumbolter even on it, and move on the slide. When supporting the slider move after a certain distance, drill can support the movement of the slider orbit, to complete a straight line on both sides of the punch, complete the drilling requirements in the area of the rules, does not need to be adjustment, and makes the work efficiency greatly. As shown in fig. 6.

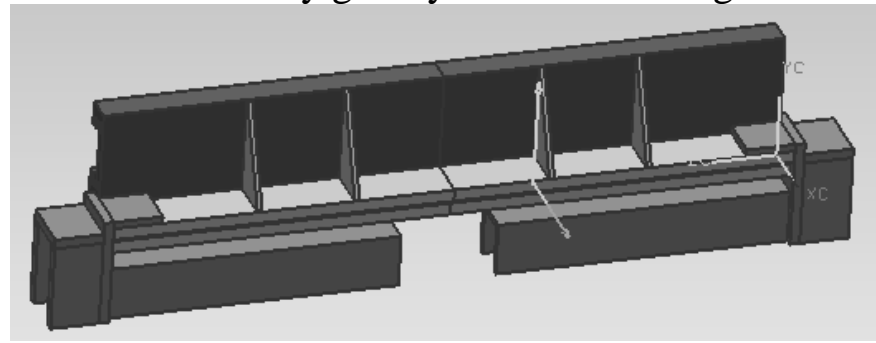

Fig.6 Support slipper

(9) Hydraulic cylinder with DG model for vehicle hydraulic cylinders. Telescopic hydraulic cylinder is placed within the support outside agencies and is connected with. When it comes to work, through the telescopic telescopic hydraulic cylinder, when the telescopic distance, make the support plate support to the ground, when drilling rig up, the downward through the leg to the ground reaction force, reduced the entire device to withstand forces. The joint of support plate and the hydraulic cylinder with spherical pair connection, when supported by the ground is not flat, so also can ensure the smooth progress of support. Supporting leg support fixed on both sides of the slider, along with the support of slider move and move. As shown in fig. 7. 


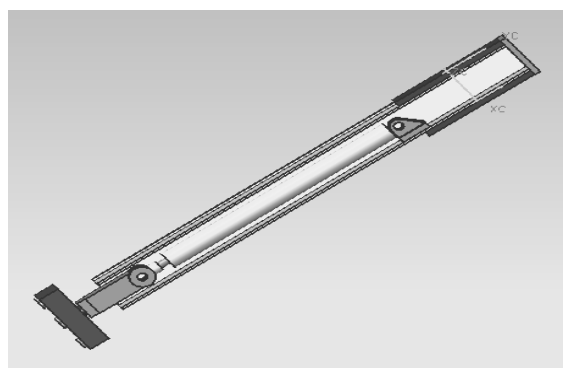

Fig.7 Outrigger of drilling rig supporting platform

\section{The 3D virtual prototype design of the robot.}

Based on 3D virtual prototype technology, combined with the requirements of automatic installation bolt robot functions, using UG as achieve its virtual prototype of 3D CAD technology. Operation according to the design of the structure size, in front of the car of the rotating arm, telescopic hydraulic cylinder 1, telescopic boom, adjust the hydraulic cylinder, bracket, drill supporting platform, telescopic hydraulic cylinder 2, support the slider, drill leg support platform key parts such as 3D modelling, and combination of assembly into working truck 3D virtual prototype, as shown in figure 7.

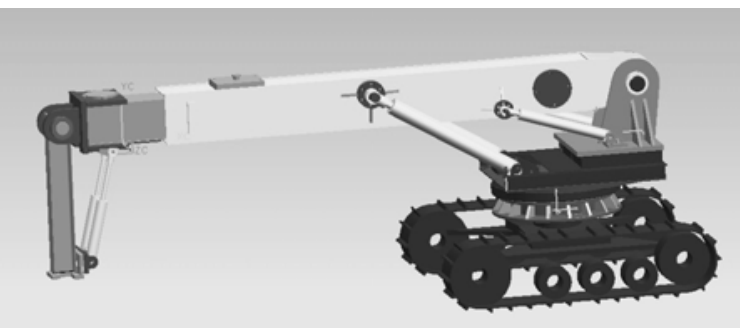

Fig. 8 Automatic installation bolt three-dimensional virtual prototype of the robot

\section{Acknowledgement}

This work was financially supported by Foundation for 2015 year of University Youth Key Teacher by the Ministry of Education of Shandong province.

\section{References}

[1] Jixin Liu, Fengju Hu, Jidai Wang and Yuanyuan Xu.Study on Design of Cable Automatic Mounting Vehicle Structure[C]. 2012 4th International Conference on Mechanical and Electronics Engineering,Weihai,China.Advanced Materials Research:Vol.588-589,p170-172.

[2] Xiangyong Guo . ZZ2-8 / 100type Mine Fully Hydraulic Drilling Machine Design Research [ J ]. Coal Mine Machinery,2011(11):5-7.

[3]Suogeng $\mathrm{Xu}$, etc. Comprehensive Tunneling Working Face Airborne Bolt Drilling Machine Design Research [J]. Journal of Coal Mine Machinery, 2007, 28 (9):5-7.

[4]Gui-ying Zhou, etc. Machine Airborne Jumbolter Research [J]. Journal of Structural Design of Coal Mine Machinery, 2008 (7):11-12.

[5] Chang-long Du. Impact Rotary Anchor Drill Design Study [J]. Journal of China University of Mining, 2002 (1):92-95.

[6]Yang Yu. Loader Crawler Travel Mechanism Analysis and Selection[J].Coal Mine Field,2009(8):242.

[7]Zaixiang Wang. About Four Arm Anchor Machine Installation Tool Clamping Device Technology Solutions [J]. Journal of Inner Mongolia, Petrochemical Industry, 2006 (9) :53-54. 